Vittorino GROSSI OSA

(Roma, Augustinianum)

\title{
LA PACE IN AGOSTINO D'IPPONA. ASPETTI ANTROPOLOGICI E SOCIALI
}

La vita di Agostino d'Ippona abbraccia il periodo del tardo antico dalla seconda metà del sec. IV al primo trentennio del sec. V (354-430) e ci può dare un'informazione non indifferente su molti problemi relativi a quell'epoca. In questa ricerca prendiamo in considerazione il problema della pace negli scritti di Agostino relativamente a tre aspetti: la pace quale legge naturale di ogni essere; la condizione esistenziale di „non-pace” dell'uomo sulla terra; l'impegno richiesto al cristiano per la pace a livello ecclesiale e civile.

1. La pace: bene di ogni essere creato. Il Vescovo d'Ippona pone la pace di ogni essere, in particolare dell'uomo, in relazione alla legge eterna della pace nella quale ogni essere viene creato. Egli se ne occupò soprattutto nell'ultima fase della polemica pelagiana, quella con Giuliano di Eclano in merito alla possibilità o meno della natura umana di poter essere creata con la concupiscenza, vale a dire con l'opposizione nel suo essere della legge della carne alla legge della mente o dello spirito. Se la pace - argomentava Agostino - domina l'intera creazione, la natura umana non può esserne stata creata esente. In virtù infatti del principio della legge eterna, la cui esigenza fondamentale è l'ordine che dà ad ogni essere la sua pace, l'uomo venne creato senza il disordine della concupiscenza ${ }^{1}$. La pace, che assegna ad ogni cosa il suo posto, viene perciò da lui definita come la ,tranquillità dell'ordine" ${ }^{2}$.

Nel libro $19^{\circ}$ della Città di Dio ${ }^{3}$ Agostino considera da vicino il problema della pace e ne enumera tuti i gradi, dal grado più infimo (la pace del corpo) a quello più elevato (la pace della città celeste), fermandosi in modo particolare

${ }^{1}$ Vedi in successione cronologica De ordine II 8, 25; De libero arbitrio I 6, 15; De civitate Dei XIX 14; cfr. G. Bardy, La paix - La loi éternelle, BAug 37, Paris 1960, 740-742, note complementari 12 e 13.

2 De civitate Dei XIX 13, 1, CCL 48, 679: „Pax omnium rerum, tranquillitas ordinis”.

${ }^{3}$ Cfr. H. Fuchs, Augustin und der antike Friedensgedanke. Untersuchungen zum 19. Buch der "Civitas Dei», 2. ed., Berlin - Zürich 1965; G. Zecchini, Il „Pius de pace” di M. Terenzio Varrone, unico scritto antico sul tema della pace, presente nel "De civitate Dei» 19 di s. Agostino, in: La pace nel mondo antico, a cura di M. Sordi, Milano 1985, 90-202. 
sulla pace del corpo e di quella dell'anima. Egli rileva come l'istintivo timore della morte sia segno della loro unione naturale e, pertanto, la loro separazione sia pena di una pace rotta, dato che l'uomo non è solo il suo corpo, né l'anima sola, ma l'unione di tutti e due ${ }^{4}$. L'aspirazione alla pace dell'essere umano pertanto è per lui un dato insopprimibile. La pace è quindi nella visione di Agostino la condizione creativa della nascita dell'uomo: con se stesso, con il creato, con Iddio. Egli scriveva a Giuliano di Eclano:

„Prima del peccato conveniva (decebat) infatti alla natura umana, la pace non la guerra" ${ }^{5}$.

e si chiedeva ancora:

„E come avrebbero potuto vivere (i progenitori) in quel luogo tanto beato senza avere la pace in se stessi, tra la mente e la carne?"6.

Per tale motivo le leggi eterne, che riuniscono nella pace gli elementi che si convengono $^{7}$, anche dopo il peccato di Adamo attendono il loro compimento nelll'uomo e nel creato. Ciò che per Agostino avverrà compiutamente con la risurrezione alla fine dei tempi ${ }^{8}$.

2. La condizione di „non-pace” dell'uomo sulla terra. L'uomo esistenziale sperimenta tuttavia la condizione di non pace sulla terra, data per Agostino dalla nascita di ogni uomo quale figlio di Adamo peccatore, che lo colloca eistenzialmente in una situazione di peccato e quindi di „,non-pace”. L'uomo nasce infatti con un'assenza di pace nella sua carne come in tutto il suo essere, una situazione che lo accompagna nell'intero arco della sua esistenza terrena". Il riposo in Dio del cuore dell'uomo, che solo può calmare la sua non-pace $\left(l^{\prime}\right.$, ,inquietum est cor nostrum"10), non appartiene perciò al mondo presente della storia. In tale ottica Agostino lesse ancora nell'anno 427 il passo del Vangelo di Lc 10, 5-7 sul filius pacis, da lui collocato in ambito di prescienza e di predestinazione divina ${ }^{11}$.

${ }^{4}$ Cfr. De civitate Dei XIII 24, 2.

Contra Iulianum III 11, 23, NBA 18, 607.

${ }^{6}$ Contra secundam Iuliani responsionem opus imperfectum 1, 71, NBA 19/1, 99.

${ }^{7}$ Cfr. l'espressione del De civitate Dei XIX 12, 3, NBA 5/3, 50: „Congrua congruis pacificantes”.

${ }^{8}$ Cfr. De civitate Dei XIII 15, NBA 5/2, 230-231: „(Solo) nella condizione della pace finale [...] la natura, sanata dall'immortalità e dall'incorruzione, non sarà più manchevole (vitiata)"

${ }^{9}$ Cfr. Augustinus, Contra secundam Iuliani responsionem opus imperfectum 6, 14, tesi fondata su Gal 5, 16-17; in Retractationes I 19, 1-2 Agostino applica la beatitudine evangelica „beati i pacifici" ( $M t$ 6) non in ordine alla guerra ma alla pace della persona singola e cioè alla sua condizione di non-pace.

${ }^{10}$ Confessiones I 1, 1, NBA 1, 4-5

11 Cfr. Augustinus, De correptione et gratia 15, 46; vedi V. Grossi, Il cristiano „filius pacis" nell'esegesi origeniana di Luca 10, 5-7, in: Origeniana sexta: Origène et la Bible. Actes di Colloquium 
Tale lettura era stata, prima di Agostino, un'opzione interpretativa di Origene in merito alla questione se Gesù avesse affidato anche a Giuda la missione di precederlo dove Lui si sarebbe recato, corredandolo come gli altri discepoli del dono della pace ${ }^{12}$. Tale interpretazione tuttavia era rimasta nell'alessandrino a livello di un dato senza altre precisazioni. Lui infatti aveva utilizzato il testo lucano principalmente per indicare nel filius pacis l'dentità del cristiano perfetto.

Negli altri autori latini, prima e contemporanei di Agostino, il testo di Luca 10, 5-7 sul filius pacis, risulta praticamente assente. Il v. 6 di Luca (pax huic domui) viene tuttavia citato da Tertulliano ${ }^{13}$ a proposito dello scambio di pace; da Gregorio di Elvira per lo Spirito Santo il quale, inviato a tutti gli uomini, se nel mondo non trova un filius pacis se ne ritorna nella Chiesa ${ }^{14}$; da Ambrogio, per il dovere dell'ospitalità ${ }^{15}$; nell'Ambrosiaster infine per indicare nel Cristo assieme al Padre la fonte della pace ${ }^{16}$.

Quanto ad Agostino egli fece riemergere, nell'anno 427, la domanda della prescienza divina che l'alessandrino aveva legato al testo lucano $(10,5-7)$, certamente in polemica con gli origeniani ${ }^{17}$. Prima di allora tale brano non aveva avuto in lui nessuna rilevanza ${ }^{18}$. Nel De correptione et gratia $^{19}$ del 427 va notato anzitutto che la lezione testuale del testo lucano è, nel medesimo paragrafo, duplice: super illum, et super illam, la pace cioè viene riferita al filius e alla domus. Il filius pacis di Agostino ha le medesime connotazioni di quelle origeniane: aderendo a Cristo con la fede si ha la pace con Dio e si diventa figli della pace. Egli tuttavia connota tali elementi nel contesto del cristiano ,,veramente figlio", un dato che può essere noto solo a Dio, non essendo possibile nel tempo della storia una conoscenza individuante il filius pacis né dall'evangelista Luca né da nessun altro. L'azione dei pastori di anime perciò - rilevava Agostino - non potendo discernere chi sia figlio della pace e chi non lo sia, è tesa ad operare verso tutti perché raggiungano la salvezza.

Origenianum Sextum (Chantilly, 30 août - 3 septembre 1993), éd. G. Dorival - A. Le Boulluec, Leuven 1995, 709-721.

12 Cfr. Origenes, In Ioannis comm. 32, 8 e 14, PG 14, 777 e 804.

13 Cfr. De oratione 26, 2, CCL 1, 273.

14 Cfr. De arca Noe 25-27, CCL 69, 153 e 174-177.

15 Cfr. Expositio Evangelii secundum Lucam 7, 62-64, PL 15, 1803-1804.

16 Cfr. Commentarius in Epistulam ad Romanos I 8, 2-3, CSEL 81/1, 21.

17 Agostino dedica nel suo De haeresibus (a. 428-430) ad Origene e agli origeniani due interi paragrafi (42 e 43). Nasce con quest'opera nelle liste ereticali occidentali il gruppo ereticale degli origeniani. Agostino li indica come ,alcuni”, che erano i monaci delle Gallie anche se non vengono nominati, vedi V. Grossi, L'origenismo latino negli scritti agostiniani. Dagli origenisti agli origenia$n i$, ,Augustinianum” 46 (2006) 51-88.

18 Si conserva una citazione in Speculum (par. 27), opera di attribuzione ma si è inclini a ritenerla del vescovo d'Ippona, e un'altra nell'Epistula 247 ad un certo Romolo che non voleva più salutarlo.

${ }^{19}$ Cfr. De correptione et gratia 15, 46. 
Il filius pacis della lettura lucana di Agostino non rappresenta più, in altri termini, il cristiano origeniano del tempo della storia, identificato appunto dalla sua visibile funzione di pace, ecclesiale e sociale, ma colui che appartiene al numero dei predestinati, a quanti cioè raggiungono la vita eterna. Nonostante queste precisazioni esegetiche e teologiche insieme l'Ipponate esplicitò enormemente l'impegno del cristiano per la pace, sia sul piano ecclesiale che su quello sociale. La condizione di non-pace della nascita di ogni uomo, data l'eredità del peccato di Adamo che, se con il battesimo viene annullata quanto a reato non viene tuttavia annullata quanto a penalità, non esime infatti il cristiano dal suo costante impegno per la pace.

3. L'impegno del cristiano per la pace. Agostino, presbitero (a. 391) e vescovo (a. 396-430) della Chiesa d'Ippona, venne a trovarsi ecclesialmente, in una Chiesa africana divisa che veniva da lontano. Tale divisione, nata agli inizi del sec. IV (a. 311), era particolarmente viva ai suoi tempi nella standardizzata divisione tra cattolici e donatisti. Socialmente poi si era al tempo delle grandi migrazioni di popoli durante le quali nel primo trentennio del sec. V i Vandali raggiunsero anche l'Africa. In tale complesso contesto ecclesiale e sociale Agostino prese posizione sull'uno e sull'altro fronte, evidenziando il particolare impegno per la pace richiesto al cristiano, nonostante la sua condizione esistenziale di non-pace.

a). Il cristiano e la pace ecclesiale. Agostino fronteggiò lo scisma donatista in Africa e conseguentemente l'impegno del cristiano per la pace ecclesiale approfondendo la comprensione di Chiesa. Egli distinse in essa due momenti: uno relativo al tempo presente della storia e uno futuro, quello escatologico.

La Chiesa del tempo presente è da lui pensata come un corpo misto (corpus permixtum) di santi e non santi, come su di un'aia si ha insieme paglia e grano ${ }^{20}$, in un campo il grano è misto alla zizzania, una rete gettata per la pesca che raccoglie ogni genere di pesci. La Chiesa, fondata su Cristo „la nostra pace”21, postula un duplice impegno dei cristiani: uno sul piano sacramentale, un altro sul piano della carità ${ }^{22}$.

Agostino, per esprimere tale duplice impegno, usò nella polemica donatista l'espressione ricorrente di ,pace di Cristo, pace della Chiesa”. Con tale dicitura egli indicava: da una parte, l'unità di tutti i cristiani (= la comunione nei sacramenti) che non si può annullare per nessun delitto ${ }^{23}$; dall'altra, l'impegno per mantenerla tramite la comunione nella carità.

\footnotetext{
${ }^{20}$ Cfr. Enarrationes in Ps. 25; Sermo 2, 5.

21 Cfr. Enarrationes in Ps. 78, 3, NBA 26, 1071.

2 Cfr. Sermo 71, 17, 28.

23 Vedi ad esempio, Epistulae 23, 5; 43, 8, 21; 53, 1, 3; 105, 2, 5; 108, 7, 20; Sermo 71.
} 
In termini di teologia sacramentaria egli distingue in un sacramento tra la sua esistenza e la sua utilità. Alla prima, corrisponde la comunione nei sacramenti; alla seconda, la comunione nella carità che conserva alla Chiesa la sua unità e la sua pace. Chi perciò - egli conclude - non ha l'utilità del sacramento, cioè la carità dataci dallo Spirito Santo, lacera l'unità e non vive nella pace cattolica. In tale ottica egli leggeva il ministero del vescovo e in particolare quello dell'apostolo Pietro, come ministero di pace per l'unità della Chiesa, ponendo tra l'altro in luce come la pace della Chiesa vada difesa anche informando il popolo di Dio delle questioni che vi si discutono ${ }^{24}$.

Nella discussione ecclesiologica con i donatisti Agostino indicò due orientamenti divenuti poi norma di ogni ecumenismo. Il primo è nel fare a meno, riguardo al passato, degli inutili rimproveri reciproci; il secondo, è trattare le controversie ecclesiali come questioni in sé, sulla base delle sacre Scritture e della fiducia nella ragione per ritrovare la verità che unisce, rinunciando pertanto ad ogni specie di violenza che può solo aumentare la divisione. Egli supplicava:

„Togliamo di mezzo gli inutili rimproveri che per ignoranza le due parti sono solite scagliarsi contro, reciprocamente. Tu non rinfacciarmi i tempi di Macario come io non ti rinfaccerò la crudeltà dei circoncellioni. Se questo fatto non ricade su di te, nemmeno l'altro ricade su di me [...]. Trattiamo della cosa in sé, con la cœrenza della ragione, con l'autorità delle sacre Scritture [...]. Chissà che il Signore, assecondando i nostri sforzi e le nostre concordi preghiere, non faccia sì che comincino a sparire dallle terre delle nostre regioni africane un obbrobrio ed un'empietà così grandi [...]. Io poi non farò questo fino a che i soldati sono qui [...] bensì dopo la loro partenza, affinché tutti gli uditori comprendano che il mio proposito non è quello che gli uomini vengano costretti, contro la loro volontà, ad aderire alla comunione di chicchessia [...]. Da parte nostra cesserà il terrore rappresentato dal potere temporale, da parte vostra cessi il terrore diffuso dalle bande dei circoncellioni”25.

Circa le disposizioni d'animo con le quali vanno affrontate le questioni ecclesiali Agostino chiedeva poi, che la controversia donatista fosse portata avanti „con santa umiltà, con pace cattolica, con carità cristiana” ${ }^{26}$. Queste buone intenzioni precipitarono allorché nel 405 l'imperatore Onorio emise a Ravenna l'editto di soppressione dei donatisti imponendo loro di tornare alla Cattolica ${ }^{27}$.

${ }^{24}$ Cfr. Retractationes I 20.

25 Epistula 23, 6-7, NBA 21/1, 126-129.

26 De baptismo contra Donatistas II 3, 4, CSEL 51, 179.

${ }^{27}$ L'editto del 405 ci è stato conservato da Agostino nell'Epistula 185, 7, 26. Per un orientamento sulla questione, vedi V. Grossi, La chiesa della carità alla prova della vicenda donatista. Nota storico-teologica, in: Associazione Teologica Italiana, De caritate Ecclesia. Il principio „amore” e la Chiesa. Atti del XI Congresso Nazionale (Trento, 9-13 settembre 1985), presentazione di L. Sartori, Padova 1987, 282-290. 
A tale legge, non chiesta dall'episcopato africano ma da esso accettata, Agostino infine acconsentì lasciandosi convincere della sua opportunità, che più tardi anche giustificò con la teoria della bontà insita nel timore di una pena. Vi fece aggiungere tuttavia due condizioni per applicarla: l'istruzione, perché essa non si tramutasse in tirannia; e la clausola che l'uso di tale legge non potesse mai giungere a comminare la pena di morte. Ne diamo i passi principali, riportati talvolta pregiudizialmente da qualche studioso come il prodromo della futura inquisizione. Ognuno li giudichi come può. Agostino scrive:

„Mi sono dovuto arrendere agli esempi messi sotto i miei occhi dai miei colleghi. Dapprima ero del parere che nessuno dovesse essere condotto per forza all'unità di Cristo, ma si dovesse agire solo con le parole, combattere con la discussione, convincere con la ragione per evitare di avere tra noi dei finti cattolici [...]. Questa mia opinione dovette però cedere di fronte a coloro che mi contraddicevano non già a parole, ma con i fatti. Mi si portava anzitutto l'esempio della mia città natale (che si era convertita in massa alla Cattolica) [...]. Non vi dico che uno possa essere buono per forza! Voglio dire che uno, per paura di un castigo che non è disposto a subire, $\mathrm{o}$ abbandona l'animosità che lo tiene lontano dalla verità conosciuta, o è costretto a conoscere la verità ignorata",28.

Nelle Ritrattazioni Agostino, parlando di un'opera antidonatista andata perduta, ci ha lasciato il suo ultimo pensiero in merito:

„Nel primo libro dissi che disapprovavo l'impiego della forza secolare per costringere gli scismatici alla comunione ecclesiale. E in verità ciò allora non mi piaceva, perché non avevo ancora sperimentato quanto male osasse la loro impunità e quanto, per cambiarli in meglio, contribuisse la diligente vigilanza della disciplina" 29 .

Agostino utilizzò ${ }^{30}$ inoltre il passo evangelico compelle intrare ( Lc 14, 23) per giustificare la costrizione in ordine al bene, fermi restando l'obbligo dell'istruzione $^{31}$ e l'esclusione della pena di morte, perché ,,altro è l'interesse di una provincia, altro quello della Chiesa" ${ }^{32}$.

Quanto alla Chiesa senza macchia né ruga, di cui parla l'apostolo Paolo (Ef $5,27)$, che nel Vangelo viene simboleggiata nella pesca miracolosa dei 153 grossi pesci ( $G v$ 21, 15-25) ottenuta gettando le reti dal lato destro della barca, essa per Agostino è „la Chiesa celeste” del futuro escatologico, la Chiesa della risurrezione, che conterà tra i suoi abitanti solo i buoni, simboleggiati appunto dal numero di 153 grossi pesci ${ }^{33}$. In essa la pace non costituirà

28 Epistula 93, 5, 16-17, NBA 21/1, 828-831.

29 Retractationes II 5, NBA 2, 158-159.

30 Cfr. Epistula 185, 6, 21-24.

${ }^{31}$ Cfr. Epistula 93, 1, 3, NBA 21/ 1, 810-811: „Se infatti ci limitassimo ad incutere timore senza istruirli, ciò apparirebbe un'ingiusta tirannia".

32 Epistulae 134, 2-4; 132, 2; 139, 2, NBA 22, 124-125; 114-115; 198-199.

33 Cfr. Augustinus, Sermo 248,3; 251,1 e 3,4; 252,7-12; Sermo 146,2; Sermo Denis 12 (= NBA XXXI/1, Sermo 147/A). 
un problema, che invece costituisce un problema-impegno per il cristiano nel tempo della storia.

b). Il cristiano e la pace sociale. Agostino, ad un anno dalla morte, e propriamente nell'anno 429 venne sollecitato ad occuparsi espressamente dell'impegno sociale del cristiano per la pace a motivo dell'invasione vandalica in Africa.

Per valutare appieno tale testimonianza vanno tenuti in considerazione due fattori: primo, il suo intervento si ebbe prima dell'invasione vandalica, rappresentando in tal modo la tradizione cristiana in merito alla guerra oltre ad essere una nobile espressione del suo animo; secondo, egli rappresentava, rispetto all'alessandrino Origene del sec. III, il cristianesimo riconciliato con l'Impero. I cristiani in altri termini, come gli altri cittadini, partecipavano ormai anche alla vita militare delle istituzioni dell'Impero. Esaminare pertanto il problema della pace nelle testimonianze cristiane dopo Costantino ci s'imbatte anzitutto in una difficoltà da tener presente. Essa è un pò storiografica - quella della pace tra cristianesimo e Impero avviata teoreticamente da Eusebio da Cesarea - ed assieme di mentalità circa la natura della Chiesa come un'associazione religiosa la quale, pur gravitando nell'ambito della società cvile, non ne sposa del tutto i parametri. Un esempio di tale complessa problematica l'abbiamo nel De civitate Dei di Agostino, scritta dopo la presa di Roma da parte di Alarico (negli anni 410-426). Tale opera segnò tra l'altro, riguardo all'impegno civico dei cristiani, un momento di revisione circa il modo della loro presenza nella società così come si era venuto affermando con la pace costantiniana. La dottrina circa l'impero e i cristiani quale si evince nella Città di Dio ha infatti un margine di disinteresse reale su tale rapporto, un dato che invece era certamente alieno al pensare del vescovo di Milano Ambrogio.

Agostino, quale pastore cristiano, s'interessò più che delle strutture, civili o religiose che fossero, della realtà esistenziale di ogni uomo il quale, al di là del suo ruolo nel saeculum, ha come suo fine duraturo la vita eterna e come movente l'amore. Egli quindi, riguardo al collocarsi del cristiano nella società, si misurò con Ambrogio in un reale confronto benché nel De civitate Dei non lo dica espressamente ${ }^{34}$.

Per l'Ipponate l'esistenza nel saeculum è uguale e comune a tutti, i fondamenti del loro collocarsi esistenziale sono tuttavia diversi (l'amor sui o l'amor $D e i)^{35}$ e, conseguentemente, lo sviluppo e il destino finale delle due aggregazioni che producono, vale a dire la Città di Dio e la città terrestre, si attestano

${ }^{34}$ Per studi più vicini ad Ambrogio su questo terreno, vedi ad es. Y.M. Duval, L'éloge de Théodose dans la Cité de Dieu (5, 26,1). Sa place, sons sens, ses sources, in RechAug 4 (1966) 135 179 (recensione di A. Van De Veer, in: REAug 13:1967, 328-329); Fr. Thélamon, L'empereur idéal d'après l'histoire écclesiastique de Rufin et de Augustin, TU 107, StPatr 10 (1970) 310-314.

35 Cfr. De civitate Dei 10, 24, NBA 5/1, 736. 
diversamente. Ciò che decide l'iter delle due societates è per Agostino la diversità dell'amore nelle scelte che si prendono nei riguardi della storia da costruire. La Chiesa visibile ha la sua „visibilità” nella partecipazione dei suoi membri alla caritas socialis e all'unità cattolica, rispetto al riconoscimento cercato dalla civitas terrena fondata sull'amore di se stessi che ha disposizione solo il tempo della storia. Il cristiano della Città di Dio vede oltre la storia e perciò non persegue tout-court tra i suoi obbiettivi primari la preminenza pubblica della Chiesa sulla politica sociale retta dall'imperatore ${ }^{36}$.

Con la pace costintiniana si fece strada in ambito cristiano la concezione di un ,imperatore cristiano”. La conseguente nuova idea di società, civile politica e religiosa, venne fatta propria in Occidente da Ambrogio, il vescovo della sede imperiale che ne tentò la realizzazione soprattutto con gli imperatori Valentiniano II e Teodosio I. Questa idea venne tuttavia relativizzata a tal punto nella Città di Dio di Agostino che in essa quasi scompaiono le categorie istituzionali di Chiesa e di Stato, anche quella di 'Chiesa cattolica', per far posto a due civitates o società che, nel tempo presente, in merito ad un duplice amore (di Dio o di se stessi) ${ }^{37}$, nascono e si sviluppano l'una nell'altra. Esse che, nel tempo della storia, non sono passibili di completa individuazione, solo alla fine si sveleranno per quel che sono, separandosi definitivamente ${ }^{38}$.

La Città di Dio tuttavia fu quindi, tra le opere di Agostino, la più attenta nel confrontarsi con Ambrogio sul terreno del rapportarsi della Chiesa con le istituzioni civili, coinvolgendo il più vasto rapporto della Chiesa con il mondo (saeculum $)^{39}$. Una riprova di tale lettura l'abbiamo nel vescovo d'Ippona quando, in occasione di un dissidio sorto tra il governatore d'Africa (il comes Bonifacio) e la corte imperiale di Ravenna, lui fece il punto sull'atteggiamento da tenersi dal cristiano in caso di guerra: circa le responsabilità dei capi militari e dei soldati nel partecipare o meno alla guerra; circa la promozione degli sforzi

${ }^{36}$ Sono indicative al riguardo le motivazioni di Agostino al magistrato Macedonio nella richiesta di grazia per alcuni condannati a morte: il munus episcopale della riconciliazione e il dovere umanitario di concedere ad ognuno la possibilità di potersi recuperare, cfr. Epistula 153 dell'anno 413/414

${ }^{37}$ Le sintesi più belle dei due amori si hanno in: Augustinus, Enarrationes in Ps. 64, 2 (a. 410 413); Enarrationes in Ps. 136, 3, 4; De Genesi ad litteram XI 15, 20 (a. 413).

38 Cfr. De civitate Dei I 35, NBA 5/1, 84-85: „La Città di Dio, nel tempo del suo pellegrinaggio nel mondo, annovera nel suo seno degli uomini uniti ad essa con i sacramenti, che tuttavia non prenderanno parte con lei al destino eterno dei santi...Le due città sono in questo secolo mescolate e intersecate l'una nell'altra, sino al giorno in cui il giudizio le separerà". Vedi anche ibidem X 32.

${ }^{39}$ Cfr. V. Grossi, Regno di Dio e città terrena. Da Costantino alla Riforma (elementi di sintesi), in: Regno di Dio e Città terrena, Torino 1979, 79-125; G. Lettieri, Il senso della storia in Agostino d'Ippona, Roma 1988. Per l'interpretazione del saeculum agostiniano di R.A. Markus (Saeculum. History and Society in the Theology of St. Augustine, Cambridge 1970), che relega la storia alla soggettività e alla sfera interiore, vedi G.Lettieri, A proposito del concetto di 'saeculum' nel De civitate Dei, „Augustinianum” 26 (1986) 481-498. 
di pace in procinto di una guerra imminente e, infine sul come intendere la pace in tempo di pace ${ }^{40}$.

Agostino distingue per il cristiano la possibilità che egli segua le norme del mondo, oppure la legge del Signore le cui esigenze sono di non uccidere gli uomini, di non partecipare alle guerre e di cercare la pace anche nelle guerre. Egli, rivolgendosi ad un cristiano che gli poneva delle domande nell'imminenza della guerra, gli rispose perentoriamente:

„Se mi chiedi un consiglio [...] conforme alla legge di Dio so perfettamente quello che ti debbo dire. I soldati di Cristo combattono non per uccidere gli uomini ma per debellare «i prìncipi, le potenze e gli spiriti del male» ${ }^{41}[\ldots]$ i doveri coniugali poi non ti impediscono...di cercare la pace perfino nelle guerre, se mai fosse necessario che tu vi prenda parte" 42 .

Al conte Dario, inviato della corte di Ravenna in Africa, spiegò poi:

„Il titolo più grande di gloria (per un capo militare) è quello di uccidere la guerra con la parola (il negoziato, le trattative) anziché uccidere gli uomini con la spada, e procurare di mantenere la pace con la pace e non con la guerra. Certo, anche quelli che combattono, se sono buoni, cercano senza dubbio la pace ma a prezzo di sangue. Tu, al contrario, sei stato inviato proprio per impedire che si tenti di spargere il sangue di chicchessia. Mentre quindi gli altri soggiacciono a un'evenienza inevitabile, tu hai una missione invidiabile" ${ }^{43}$.

La risposta del conte Dario ad Agostino ci dà ancora qualche elemento circa la promozione della pace da parte di un cristiano del tardoantico. Lui scrisse rivolto ad Agostino:

„Tu, dopo Dio Salvatore, sei il mio salvatore...Dio faccia sì che io risponda all'opinione che hai di me...Tu affermi che io uccido la guerra con la forza della parola (= la trattativa). A questo punto il mio animo è uscito, per così dire, dalle tenebre dei suoi pensieri [...]. Infatti se non siamo riusciti a spegnere la guerra, l'abbiamo per lo meno differita e, con l'aiuto di Dio, sovrano di tutti, sono state mitigate le sciagure" $" 44$.

La posizione di Agostino, ad un anno dalla sua morte, acquista tanto più rilievo quando si pensa che lui apparteneva alla cultura della fase storica dei „tempi cristiani”, quando la fede non solo si sforzava di animare le strutture umane ma era tentata anche di qualificarle come tali, cioè come cristiane.

${ }^{40}$ L'epistolario legato a tale dissidio (Epp. 220; 229; 230; 231) è da datarsi, secondo Vera Paronetto (Nota sulla datazione dell'epistolario agostiniano: epp. 220; 229; 230; 231, „Augustinianum" 14:1974, 363-367), prima della invasione dei Vandali in Africa avvenuta nel 429,.

41 Epistula 220, 9, NBA 23, 630-631.

42 Ibidem 220, 12, NBA 23, 632-633.

43 Epistula 229, 2, NBA 23, 708-711.

44 Epistula 230, 2-3, NBA 23, 714-715. 
La questione che agli inizi del sec. III poneva Tertulliano: „Se il servizio militare sia in tutto compatibile con l'essere cristiani" 45 , al tempo di Agostino non si poneva più. Che anzi le proibizioni contenute ad esempio nella Traditio apostolica di non consentire al soldato cristiano di uccidere, al giudice di condannare a morte e ad un catecumeno di potersi arruolare nell'esercito, erano state rielaborate dalle Constitutiones Apostolicae nel contesto di partecipazione alla vita dell'Impero, anche di quella militare. Al soldato si chiedeva, nella linea della risposta di Giovanni Battista data ai soldati del suo tempo che ricorrrevano a lui, di accontentarsi della paga e di non agire ingiustamente. In tale nuovo contesto si giunse anche a sanzioni canoniche contro gli obbiettori di coscienza, ritenuti ormai dei disertori ${ }^{46}$.

Da Origene in poi tuttavia molti cristiani, mentalizzati dall'idea del cammino unitario dell'umanità verso una fede comune, crearono nuovi indirizzi storiografici circa la necessità della guerra che incisero nella valutazione di come rapportarsi di fronte alle stesse invasioni barbariche. Esse andavano giudicate non solo a livello di un danno subìto ma anche come possibilità di far conoscere ai nuovi popoli la fede cristiana ${ }^{47}$. Sforzi pratici, di mediazioni per l'incontro tra vecchi e nuovi popoli, furono la figura del santo pellegrino che accompagnava i popoli invasori mediando umanità tra loro e gli invasi. San Severino del Norico, sepolto a Frattamaggiore presso Napoli, ne costituisce una delle testimonianze più note.

4. Le conclusioni. Per il cristiano Agostino la pace è anzitutto un bene del cuore $^{48}$ di cui Cristo è l'autore e l'istitutore ${ }^{49}$. Per lui amarla è già possederla anche se nella speranza. Ed essa, simile al miracolo della moltiplicazione dei

45 De corona 11, 1, CCL 2, 1056: „, an in totum christianis militia conveniat”.

46 Il concilio di Arles del 314 sanzionava ad esempio la scomunica per i disertori, cfr. Concilium Arelatense, can. 3, Mansi II 471: „De his qui arma proiciunt in pace, placuit abstineri a communione".

47 Origene ribatteva al pagano Celso: „Gli estranei alla fede ci chiedono di combattere per il bene comune uccidendo uomini (concludeva poi con la proposta cristiana di una grande utopia, quella d'incontrarsi un giorno, nella fede in Gesù Cristo, oltre che con i pagani del mondo ellenistico-romano anche con i barbari) [...]. Se tutti gli uomini facessero come me (cristiano), è evidente che i barbari, convertiti anch'essi al Verbo di Dio, sarebbero sottomessi alle leggi e si civilizzerebbero, cesserebbero cioè di essere barbari [...]. Sì, un giorno, solo il culto cristiano sarà in vigore perché il Logos non cessa di conquistare sempre altre anime. Noi cristiani non brandiamo più la spada contro qualsivoglia popolo, né ci esercitiamo a fare la guerra; noi siamo divenuti figli di pace mediante Gesù Cristo, Lui il nostro condottiero". L'idea d'incontro tra popoli con le migrazioni è anche presente in filigrana nelle Storie dello spagnolo Orosio, scritte su suggerimento di Agostino, vedi V. Grossi, La pace nei Padri della Chiesa. Origene-Agostino-Bernardo, in: La Pace sfida all'università cattolica. Atti del Simposio...anno internazionale della Pace 1986, Roma 1988, 535-546.

48 Cfr. Sermo 357, 2.

49 Cfr. Sermo 358, 6. 
pani, cresce in proporzione di coloro che la posseggono ${ }^{50}$. Chi l'ama veramente ama perciò anche i nemici della pace ${ }^{51}$, chi infatti la possiede risponde pacificamente ad ogni litigioso ${ }^{52}$. Chi invoca Dio con un'unica voce, e cioè con il nome di „Padre”, dà già il suo impegno per perseguire un'unica pace ${ }^{53}$, quella di Cristo per la quale impegna anche il proprio onore ${ }^{54}$. La pace è una sola, essa perciò non soffre divisione e di essa - ingiunge Agostino - bisogna sempre parlare come della carità ${ }^{55}$. La pace, da parte sua, è tuttavia vera ed eterna quando si diventa veramente cioè definitivamente figli di Dio ${ }^{56}$.

Il vescovo d'Ippona vede tuttavia il problema della pace in una duplice accezione: personale e di convivenza umana. Nel primo caso egli colloca il problema, per lui connesso a quello della felicità e della gioia, oltre le frontiere del tempo, nel raggiungimento del Cristo Risorto. Egli lo evidenzia con i paragoni di unione che esiste tra la natura umana e divina in Cristo, tra Cristo e la Chiesa, tra un uomo e una donna uniti in matrimonio ${ }^{57}$, tra l'anima e il corpo ${ }^{58}$. E' la pace dell'uomo con Dio: „Tu mi toccasti, scrive nelle Confessioni, e arsi della tua pace” ${ }^{\prime \prime}$, perché Dio è per l'uomo „Colui dal quale allontanarsi è cadere, al quale ritornare è risorgere, nel quale rimanere è costruirsi solidamente" ${ }^{" 60}$.

Nonostante la sua visione escatologica della pace l'Ipponate esplicitò enormemente l'impegno del cristiano per la pace, sia sul piano ecclesiale che su quello sociale. Agostino infatti ha lasciato indicazioni concrete per fronteggiare l'eplodere di quelle tensioni che noi chiamiamo guerra.

Sul piano personale coniò l'ultimo principio etico universale trasmessoci dall'antichità: la capacità di discernere e di gestire l'uti e il frui, vale a dire ciò che appartiene alla natura delle cose che è quello di essere usate (uti), e ciò che è proprio della natura spirituale che è l'essere goduta (frui). Tale principio fece un tutt'uno con la sua spiritualità, articolata nel vocabolario unitario di cor misericordia- amicitia $^{61}$.

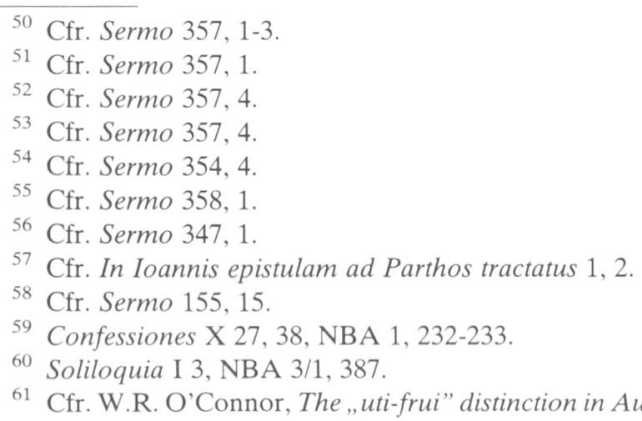
R.J. Teske, Love of Neighbour in S.A., in: Atti del Congresso Internazionale su S.A. nel XVI centenario della conversione, III, Roma 1987, 81-102; V. Grossi, Valenza antropologica della misericordia in s.Agostino, in: Dives in misericordia, Roma 1981, 189-195; V. Grossi, Il "Cor" nella spiritualità di S. Agostino, in: L'antropologia dei maestri spirituali, Roma 1991, 125-142. 
La guerra, nel contesto dell'uti e del frui, stravolgendo la funzione dell'uti col porla al posto del frui, è frutto di un uomo che non discerne e di conseguenza non gestisce adeguatamente la natura degli esseri creati. La promozione cristiana per la pace si pone nel formare un uomo che sia capace di gestire l'uti e il frui. Agostino affrontò poi direttamente anche il problema di come fronteggiare il problema della guerra, considerato uno stravolgimento di tale distinzione e conseguentemente della loro diversa funzione.

L'impegno sociale del cristiano è duplice, riguardo alla pace della Chiesa e alla pace del mondo. Nel primo caso il suo impegno si commisura al suo amore per la Chiesa di Cristo, la cui unità non si può frangere, pena la perdita del dono della carità; nel secondo caso si commisura alle sue possibilità civili, in ogni condizione della sua vita, da soldato o meno.

La Chiesa post-costantiniana acquisì in un primo momento il riconoscimento della propria indipendenza, basandosi sulla distinzione di poteri diversi, che ottenne ufficialmente con Valentiniano II; passò poi ad affermare, nei riguardi dell'impero, una sua preminenza per la natura del potere che essa rappresenta. Il mediatore di quest'ultimo passaggio fu Ambrogio di Milano. Esso si consolidò in occasione di noti avvenimenti pubblici accaduti nel sec. IV: la statua della Vittoria riportata dal prefetto di Roma Simmaco nel senato che fu fortemente constestata da Ambrogio ${ }^{62}$, il rifiuto di cedere agli ariani la basilica Porziana $^{63}$, la contestazione per la ricostruzione della sinagoga di Callinico senza previa consultazione dei vescovi ${ }^{64}$, l'imposizione all'imperatore di fare penitenza pubblica per il massacro di Tessalonica ${ }^{65}$.

Per Agostino il rapporto si poneva diversamente. Se il governo civile si occupa di un determinato Stato, la Chiesa, società vasta quanto la famiglia umana, non si preoccupa dei regimi umani che sono mutevoli, ma ad essi adatta la sua presenza ${ }^{66}$. Il vescovo d'Ippona parla quindi, più che di due

62 Si volle sottomettere la causa a Teodosio, ma Ambrogio obbiettò che si trattava di una causa religiosa e quindi di competenza ecclesiastica, l'imperatore stesso pertanto era suddito di Dio e della fede, cfr. P. Meloni, Il rapporto tra impegno politico e fede religiosa in Simmaco e Ambrogio, „Sandalion” 1 (1978) 153-169.

${ }^{63}$ Le motivazioni di Ambrogio furono due: La cura delle chiese spetta al vescovo (Epistula 76, 9, Maur. 20: , ad imperatorem palatia pertinent, ad sacerdotem ecclesiae”); l'imperatore è nella Chiesa non al di sopra di essa (Sermo contra Auxentium: „Imperator enim intra Ecclesiam, non supra Ecclesiam est"). Sulle strutture sociali del tempo nell'opera di Ambrogio, vedi M. Adriaans, „Omnibus rebus ordo". Vorstellungen über die Gesellschaftsstruktur im Werk des Bischofs Ambrosius von Mailand, Engelsbach 1995.

${ }^{64}$ Cfr. Epistula 1, 25 extra, Maur. 41.

65 Cfr. Epistula 11, 6 extra, Maur. 51; Paulinus Nolanus, Vita Ambrosii 24, in: De civitate Dei $\mathrm{V}, 24-26$.

${ }^{66}$ Cfr. De moribus Ecclesiae catholicae I 48, NBA 13/1, 79: „O Chiesa, tu unisci i cittadini ai cittadini e gli uomini, ricordandosi dei progenitori, tu li raduni tra loro [...]. (Essa) è più di una società [...] è una fraternità". Vedi anche: ibidem I 65; De utilitate credendi 16, 34-35, NBA 6/1, 232 239. La problematica moderna del rapporto in qualche modo necessario che intercorre tra le 
autorità da concordare, Chiesa e impero, dell'apporto del cristiano alla civitas terrestre come partecipazione alla storia comune del mondo, non quindi come realizzazione di una politica o di un modello di governo ${ }^{67}$.

Dobbiamo anche rilevare che la riflessione di Agostino sul tempo ${ }^{68}$ implicava la fine della concezione ufficiale della storia, quale veniva registrata negli Annales della Repubblica romana prima e dell'impero poi, quella cioè gestita dai Cesari, dalle grandi famiglie senatoriali e, da ultimo, dai condottieri dei nuovi popoli invasori ${ }^{69}$. Le volontà dei Cesari si potevano, per Agostino, solo accomunare alle volontà di tutti, non sostituirle. Il vescovo d'Ippona, che nelle Confessioni aveva portato la concezione del tempo a misura dell'uomo, dopo l'invasione di Roma da parte delle truppe di Alarico indicava negli uomini stessi il livello dei tempi che scorrono nella storia:

„Noi siamo i tempi: quali noi siamo, tali sono i tempi [...] un mondo cattivo lo fanno gli uomini cattivi"

„i cristiani fanno tempi cristiani” ${ }^{\text {, }}$;

strutture umane e la loro funzione, ad es. la giustizia, non fu presente ad Agostino come non lo era a quelli del suo tempo. Per tale motivo non ci si preoccupava della modalità dei regimi politici ma dell'onestà delle persone che li gestivano. Vedi Augustinus, De civitate Dei XIX 17, NBA 5/3, 63: „La città celeste sa che le istituzioni sono varie e mutevoli: le leggi differenti, contraddittorie, spesso sospette di essere portatrici di dubbia giustizia; come vari sono i costumi e gli stessi governi che non usano sempre i medesimi metodi, ma essa accetta tale originalità dello stato e mette tutto in opera per mantenervi la pace terrestre". Per la diversa modalità nel governare: l'impero con le armi, la Chiesa con la carità e sanzioni di ordine spirituale; vedi anche Augustinus, Contra Cresconium III 33.

${ }^{67}$ Con il De officiis Ambrogio aveva tentato una codificazione della morale cristiana che mettesse in evidenza le virtù civiche, cfr. R. Thamin, S.Ambroise et la morale chrétienne au IV siècle. Étude comparée des traités „Des devoirs” de Ciceron et de S. Ambroise, Paris 1985; Agostino teorizzò che il cristiano è il miglior cittadino perché le virtù civiche sono un'esigenza della fede cristiana, cfr. Epistula 138, dove viene sviluppato il tema che lo stato ricava salute e grandezza dai cittadini cristiani.

${ }^{68}$ Cfr. Confessiones XI dell'anno 400 e Sermo 80 e 81 del 410/411.

${ }^{69}$ Vedi tra gli altri, Y.M. Duval, Les métamorphes de l'historiographie aux IVe et Ve siècles. Actes du VII congrès de la F.I.E.C., II, Budapest 1983.

70 Sermo 80, 8, NBA 30/1, 592-593.

71 Sermo 81, 7, NBA 30/1, 604-605; cfr. anche Sermo 80, 8, NBA 30/1, 592: „Nos sumus tempora: quales sumus, talia sunt tempora [...] malum mundum mali homines faciunt"; Sermo 81, 7-8, NBA 30/1, 606: „Ecce quid faciunt tempora christiana... Ruit mundus, senescit...noli timere”. Sul rapporto dei cristiani con il mondo, vedi l'intero Sermo 81 e G. Madec, „Tempora Christiana". Expression du triomphalisme chrétien ou récrimination païenne?, in: C.P. Mayer (ed.), Festschrift A. Zumkeller, Würzburg 1975, 112-136. I sermoni 80 e 81, che sono datati da Verbraken tra il 410-411, sono certamente un'esplicitazione della riflessione sul tempo contenuta in Confessiones libro XI, cfr. V. Peri, Christianitas: criteri di una periodizzazione, „Studium” 80 (1984) 339-351 dove si rileva che Christianitas fu l'espressione medievale dopo quella patristica di Tempora christiana. 
„Dio, nella vecchiaia del mondo, quando ogni cosa cadeva a pezzi, inviò il Cristo per rifarlo. Quando ogni cosa invecchia, Cristo viene per rinnovellare anche te. Il mondo è come l'uomo: nasce, cresce, invecchia [...] non restare attaccato a questo vegliardo che è il mondo. Non rifiutare di ringiovanirti nel Cristo che ti dice: il mondo perisce, invecchia, scompare, esso é travagliato dal tarlo della vecchiaia. $\mathrm{Tu}$, non avere paura! La tua giovinezza si rinnovellerà come quella dell'aquila",72.

Quanto al fronteggiare la non-pace ovvero una guerra Agostino, dalla convinzione che la società è mossa dalla brama di possesso e dalla libido dominandi, fa spingere il cristiano ad impegnarsi non tanto a promuovere la giustizia con l'aggressione diretta, quanto a restringere l'avanzata dell'ingiustizia, facendo paradossalmente una guerra di pace perché vi sia pace, una pace cioè in cui essa è lo scopo della guerra ${ }^{73}$.

In quest'ultimo senso in Agostino si parla anche di una necessità della guerra, e di guerra giusta. Anche in tal caso tuttavia per lui la guerra è da avversare. Agostino fu soprattutto molto critico nelle guerre dei Romani che erano compiacenti di molte aggressioni avvallate come interventi giusti (è questo il significato del famoso dialogo tra Alessandro Magno e un pirata, riportato nella Città di Dio $)^{74}$.

Lo stesso concetto espresso con l'espressione di „guerra di difesa” non rende giustizia ad Agostino nel capire il suo concetto di "guerra giusta", concetto -egli precisava- che non si può mai addebitare a $\mathrm{Dio}^{75}$. Per tale motivo il vescovo d'Ippona non può essere invocato come patrono della „guerra santa”, che fu un'idea medievale ${ }^{76}$. Se Agostino consideri la giusta guerra in ambedue i contendenti, è una discussione che si ha tra gli studiosi, si tratta comunque di categorie che in lui vanno lette nel contesto delle relative forme di giustizia. Altrimenti, per ogni uomo saggio la guerra è da evitare, scrive Agostino ${ }^{77}$. La guerra, in altri termini, è l'ultima risorsa per un uomo di pace $^{78}$. Nell'ottica dell'ultima risorsa a disposizione egli, per scoraggiare il commercio degli schiavi, intervenne suggerendo, come prevenzione, anche la pena di morte per tali commercianti ${ }^{79}$.

72 Sermo 81, 8, NBA 30/1, 606-609.

73 Cfr. De civitate Dei XIX 12-13.

74 In Heptateuchum 6, 10 si ha la definizione intesa comunemente ,definiri solent” come "guerra giusta", dalla quale Agostino prende le distanze. Per tale motivo Agostino evita di proposito di precisare ,la giusta causa”.

75 Cfr. Contra Faustum XXII 71-72.

76 Cfr. W. LeRoy, The Just War and the Crusade: Antitheses or Analogies?, „Monist” 57 (1973) 584-594.

77 Cfr. De civitate Dei IV 15; XIX 7.

78 Cfr. Epistula 189, 6.

79 Epistula 10* (ed. Divjak), BAug 46, 466-474. 